\title{
Nuclear and mitochondrial genome responses in HeLa cells treated with inhibitors of mitochondrial DNA expression
}

\author{
Janusz Piechota ${ }^{1 \star}$, Roman Szczęsny ${ }^{2}$, Kamila Wolanin ${ }^{1}$, Aleksander Chlebowski ${ }^{1}$ and \\ Ewa Bartnik ${ }^{1,2 \otimes}$ \\ ${ }^{1}$ Department of Genetics and Biotechnology, Warsaw University, and ${ }^{2}$ Institute of Biochemistry and Biophysics,

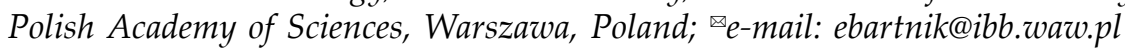

Received: 08 June, 2006; revised: 14 August, 2006; accepted:14 August, 2006

available on-line: 02 September, 2006

\begin{abstract}
The influence of mutations in the mitochondrial DNA (mtDNA) on the bioenergetic metabolism of the cell is still poorly understood. Many of the mutations in the mtDNA affect the expression of the mitochondrial genome. Investigations on cells from patients are not easy, especially as the mitochondrial DNA is heteroplasmic and this state is changed in culture. Moreover, the nuclear background and the mitochondrial haplotype may affect the behaviour of cells. Transfer of patient mitochondria to rho zero cell lines is also not optimal as these cells in general have many nuclear changes which may also affect cell behaviour. Thus, we decided to use inhibitors of mitochondrial genome expression, such as thiamphenicol, ethidium bromide and dideoxycytidine to investigate the bioenergetic metabolism of HeLa cells. We found that oxidative phosphorylation and glycolysis participate equally in ATP production in HeLa cells and that decreased activity of the respiratory chain leads to increased glycolysis and the reduction of cell growth. Insufficient ATP production in the oxidative phosphorylation process was not compensated by increased proliferation of the mitochondria. However, we were able to show that there are some mechanisms compensating limited expression of the mitochondrial genome within the mitochondria. Experiments with dideoxycytidine revealed that 10 -fold decrease of the mtDNA copy number resulted in almost normal activity of cytochrome $c$ oxidase. We found that mtDNA depletion is compensated mostly on the level of RNA metabolism in the mitochondria. Thus, our results are in agreement with the hypothesis that transcription initiation rather than mtDNA copy number is a rate limiting factor for expression of the mitochondrial genome.
\end{abstract}

Keywords: HeLa, mitochondrial biogenesis, mtDNA depletion, thiamphenicol, ethidium bromide, dideoxycytidine

\section{INTRODUCTION}

Mitochondria play a central role in the metabolism of eukaryotic cells. One of the most important functions of mitochondria is ATP synthesis in the oxidative phosphorylation process in which five multisubunit complexes forming the respiratory chain are involved (Rotig \& Munnich, 2003). Four complexes are responsible for generation of the proton gradient across the inner mitochondrial membrane. The fifth complex, $\mathrm{F}_{0} \mathrm{~F}_{1}$-ATPase, uses this gradient for ATP generation. The respiratory chain complexes are composed of over 80 proteins encoded mainly in the nucleus, though 13 subunits are encoded in the mitochondrial DNA. The 24 remaining mitochondrial genes ( 2 rRNAs and 22 tRNAs) are involved in the synthesis of these subunits.

Thus mutations leading to dysfunction of the respiratory chain complexes may occur in two different genomes. Genetic disorders of the respiratory chain have been linked to almost two hundred point mutations in the mitochondrial genome and over

$\star$ Present address: Department of Biotechnology, University of Wrocław, Wrocław, Poland.

Abbreviations: ANT, adenine nucleotide translocator; CS, citrate synthase; ddC, dideoxycytidine; GAPD, glyceraldehyde3-phosphate dehydrogenase; mtDNA, mitochondrial DNA; NRF, nuclear respiratory factor; PBS, phosphate-buffered saline; PGC, peroxisome proliferator-activated receptor gamma coactivator; PRC, Pgc-1-related coactivator; RRFs, red ragged fibers; TFAM, mitochondrial transcription factor A; TFB1M and TFB2M, mitochondrial transcription factors B1 and B2. 
one hundred large-scale deletions of mitochondrial DNA (mtDNA) collected in the MITOMAP database (Brandon et al., 2004). The relationship between mitochondrial disorders and mutations has been documented for several dozen of the nuclear genes encoding proteins directly or indirectly involved in the biogenesis of the respiratory chain complexes (Rotig \& Munnich, 2003). It was estimated that respiratory chain disorders (also called OXPHOS disorders) affect at least 1 in 8000 individuals, placing them in the group of the most frequent metabolic inborn errors (Skladal et al., 2003).

Mutations in both mitochondrial and nuclear genes resulting in defects in the respiratory chain complexes lead predominantly to insufficient ATP production. However, other effects, such as increased production of reactive oxygen species or sensitization to apoptotic signals may play an important role in the pathology of respiratory chain disorders (McKenzie et al., 2004). Mechanisms compensating insufficient production of ATP are largely unknown. One of the possibilities is increased glycolysis rate, as the ATP produced in the glycolytic pathway is the only alternative source of energy. Reduced $\mathrm{NAD}^{+}$produced by the glycolytic pathway is recovered by lactate dehydrogenase, leading to production of lactate from pyruvate. In fact, lactic acidemia (increased lactate concentration in blood plasma) is a symptom frequently observed in patients diagnosed with mitochondrial disorders (Leonard \& Schapira, 2000). Increased proliferation of mitochondria is the other possibility for compensation of ATP deficiency. This mechanism is supported by the presence of red ragged fibers (RRFs) in muscle biopsies of patients harbouring mutations in mitochondrial DNA (Leonard \& Schapira, 2000). RRFs are muscle fibers with accumulation of abnormal mitochondria in the subsarcolemmal area (Dang \& Semenza, 1999). RRFs have been shown to possess extremely high levels of mutated mtDNA in comparison with surrounding fibers (Petruzzella et al., 1994; Ozawa et al., 1998). Additionally, Heddi et al. $(1993,1999)$ observed some aspects of enhanced mitochondrial biogenesis in muscle biopsies of MERRF (myoclonic epilepsy and ragged red muscle fibers) and MELAS (mitochondrial encephalomyopathy, lactic acidosis, and stroke-like episodes) patients with diagnosed mutations in the mitochondrial genome. The samples exhibited increased levels of mitochondrial transcripts as well as increased levels of transcripts of nuclear genes encoding proteins involved in energy metabolism, e.g. ATP synthase $\beta$ subunit, ANT1 and ANT2 (adenine nucleotide translocator 1 and 2), hexokinase, and E1 $\alpha$ subunit of pyruvate dehydrogenase. Elevated levels of these transcripts have been correlated with high levels of mutated DNA. Thus, Hed- di et al. (1999) suggested that defects in oxidative phosphorylation may be compensated in human tissues by stimulation of mitochondrial biogenesis.

Mitochondrial proliferation is a tightly controlled process regulated by several recently identified transcription factors, including nuclear respiratory factors 1 and 2 (NRF-1 and NRF-2), PGC-1 (peroxisome proliferator-activated receptor gamma coactivator 1) and PRC (Pgc-1-related coactivator). Interactions between these factors create a complex system controlling synthesis of the mitochondrial proteins encoded in the nucleus. This system also controls expression of the mitochondrial genome through mitochondrial transcription factor A (TFAM) and mitochondrial transcription factors B1 and B2 (TFB1M and TFB2M). Nuclear control of mitochondrial biogenesis has been well characterised in reviews by Goffart and Wiesner (2003) or Scarpulla (1997; 2002; 2005). Mitochondrial biogenesis is regulated by several intracellular (e.g. calcium ion concentration $(\mathrm{Wu}$ et al., 2002), nitric oxide (Nisoli et al., 2003) or ATP/ AMP ratio (Zong et al., 2002)) and extracellular (e.g. thyroid hormone (Pillar \& Seitz, 1997), glucocorticoid hormones (Tsiriyotis et al., 1997), insulin (Stump et al., 2003)) factors.

Although induction of mitochondrial proliferation in tissues of patients with defects in mitochondrial DNA disorders is well documented, the mechanism of this induction is still not understood. Finding the signals inducing mitochondrial biogenesis would be very important in understanding the pathology of mtDNA diseases. Since mitochondrial biogenesis is governed by a complicated system regulated by various extracellular and intracellular signals, there is a need for a careful examination of how decreased expression of the mitochondrial genome influences the biogenesis of the mitochondria and the respiratory chain complexes. We attempted to answer this question by utilization of inhibitors affecting various steps of mitochondrial genome expression. In our investigations we used thiamphenicol, a specific inhibitor of mitochondrial translation and ethidium bromide, an inhibitor of mitochondrial transcription and replication processes. We found that pronounced, but short-term inhibition of mtDNA expression in HeLa cells by both drugs did not induce enhanced mitochodrial proliferation. However, additional experiments with cells partially depleted of mitochondrial DNA revealed possible compensatory mechanisms in the mitochondria at the level of mitochondrial RNA metabolism.

Investigations on cells from patients are not easy, especially as the mitochondrial DNA is heteroplasmic and this state is changed in culture. Moreover, the nuclear background and the mitochondrial haplotype may affect the behaviour of cells. Transfer 
of patient mitochondria to rho zero cell lines is also not optimal as these cells in general have many nuclear changes which may also affect cell behaviour (Singh et al., 2005). We thus decided to analyse the effects of several inhibitors on mitochondrial respiration and replication and on the levels of mitochondrial transcripts and nuclear and mitochondrial-encoded proteins.

\section{MATERIALS AND METHODS}

Cell culture and inhibitors. HeLa cells were grown in DMEM medium supplemented with $10 \%$ fetal bovine serum, $2 \mathrm{mM}$ glutamine, $1 \mathrm{mM}$ pyruvate and $50 \mu \mathrm{g} / \mathrm{ml}$ uridine, in conditions of $37^{\circ} \mathrm{C}, 5 \% \mathrm{CO}_{2}$ and $100 \%$ humidity. Tissue culture media, supplements, fetal bovine serum and inhibitors were from Sigma. Plasticware was from Sarstedt. Ethidium bromide was used at a concentration of $50 \mathrm{ng} / \mathrm{ml}$. The concentration of dideoxycytidine in the medium was $20 \mu \mathrm{M}$. The working solution of thiamphenicol was prepared at $50 \mathrm{mg} / \mathrm{ml}$ in DMSO as a diluent. The final thiamphenicol concentration in the cell culture medium was $50 \mu \mathrm{g} / \mathrm{ml}$. In this case, cells pretreated for $48 \mathrm{~h}$ with $0.1 \%$ DMSO were used as a control.

Western blot analysis. Western blot analysis was as described by Sambrook \& Russel (2001). Briefly, cell samples were suspended in Laemmli buffer and sonicated $(2 \times 15 \mathrm{~s})$. Aliquots $(15-30 \mu \mathrm{g})$ of the samples were loaded on SDS/PAGE gels. Electrophoresed samples were transferred to Protran nitrocellulose filters (Schleicher \& Schuell Bioscience) by semi-dry transfer and blocked by incubation in $10 \%$ non-fat milk in TBS-T. Primary antibodies were used as follows: anti-GAPD (glyceraldehyde-3-phosphate dehydrogenase) antibodies (ABCAM), anti-cytochrome $c$ antibodies (Santa Cruz Biotechnology), anti-COX2 and anti-COX5a antibodies (cytochrome $c$ oxidase subunits 2 and 5a) (Molecular Probes), anti-Hsp60 antibodies (Sigma). Secondary antibodies (Sigma) conjugated with horseradish peroxidase were visualised by ECL reagent.

mtDNA quantification. Total DNA was isolated using DNA Tissue Kit (Qiagen). mtDNA levels were analysed by real-time PCR using QuantiTect SYBR Green PCR Kit (Qiagen). Previously described primers encompassing the ND3 gene were used for quantification of the mtDNA molecules (Piechota et al., 2006). Primers for the B2M ( $\beta$-2-microtubulin) nuclear gene described by Vandesompele et al. (2002) were used for standardization.

Analysis of transcript steady-state levels. Steady-state levels of the mitochondrial COX2 mRNA as well as NRF-1 and TFAM transcripts were analysed using real-time PCR technique. RNA was isolated using TRI Reagent (Sigma) according to the man- ufacturer's instructions. DNA-free RNA samples were prepared using Deoxyribonuclease I (Fermentas). Reverse transcription was performed using Omniscript RT Kit (Qiagen) and oligo-(dT) 18 primer. Real-time PCR reaction was performed on LightCycler (Roche) using QuantiTect SYBR Green PCR Kit (Qiagen). Primer sequences were as follows: TFAM-R: GATGAGTTCTGCCTGCTTTATG, TFAM-F: GGACTTCTGCCAGCATAATACT, NRF1-R: TTTCCTCAGGTGAGTCCAGCAG, NRF1-F: TTTCCTCAGGTGAGTCCAGCAG. Primer sequences for COX2 analysis have already been published (Piechota et al., 2006).

Steady-state levels of $16 \mathrm{~S}$ rRNA were assayed using Northern blot analysis. Aliquots of $5 \mu \mathrm{g}$ RNA were electrophoresed in 1\% denaturing formaldehyde-agarose gels and transferred to Hybond-N filters (Amersham International), following the manufacturer's instructions. Filters were prehybridized in PerfectHyb ${ }^{\text {TM }}$ Plus hybridization buffer (Sigma). The PCR product encompassing the 1756-2444 mtDNA fragment was used as a probe for 16S rRNA. Northern blots were standardized on 7SL RNA. The PCR product used as a probe was amplified with primers 7SL-F: TCGGGTGTCCGCACTAAGTT and 7SL-R: TGGCTATTCACAGGCGCGAT. The relative steadystate levels of the RNAs were detected by densitometry and quantified using ImageQuant software.

Lactate and pyruvate production. The protocol used for analysis of lactate and pyruvate production was based on the protocol for fibroblasts described by Robinson (1996) with slight modifications. HeLa cells were harvested and washed twice with PBS. Approximately $10^{7}$ cells were resuspended in $1 \mathrm{ml} \mathrm{PBS}$ and incubated at $37^{\circ} \mathrm{C}$ for $15 \mathrm{~min}$. Then cells were centrifuged, resuspended in $1.5 \mathrm{ml}$ PBS plus $25 \mathrm{mM}$ glucose and incubated at $37^{\circ} \mathrm{C}$ with occasional shaking in order to avoid sedimentation of the cells on the bottom of the tube. After $1 \mathrm{~h}$ incubation cells were centrifuged $(5000 \times g$, $3 \mathrm{~min})$. Cell pellets were used for the measurement of the protein content, and supernatants for analysis of lactate and pyruvate concentrations. Lactate concentration was analysed using Lactate Reaction Kit (Sigma) according to the manufacturer's instructions. Pyruvate concentration was determined spectophotometrically at $340 \mathrm{~nm}$ by a standard method utilizing lactate dehydrogenase (Sigma) and NADH.

Enzyme activities. Biochemical assays of the cytochrome $c$ oxidase and citrate synthase activities in the digitonin-treated cells were carried out essentially as described by Darley-Usmar et al. (1987). $5 \times 10^{6} \mathrm{HeLa}$ cells were taken for preparation of the mitochondria-enriched fraction by digitonin treatment. Cytochrome $c$ from bovine heart used in the cytochrome $c$ oxidase activity assay was obtained from Sigma. Measurements of the enzymatic activities were carried out at room temperature. 


\section{RESULTS} Thiamphenicol and ethidium bromide disrupt ex-
pression of the mitochondrial genome

Thiamphenicol, a derivative of chloramphenicol is an inhibitor of the mitochondrial translation system (Kroon, 1965). Ethidium bromide was shown to inhibit mitochondrial transcription and replication systems (Seidel-Rogol \& Shadel, 2002). Thus, both inhibitors disrupt expression of the mitochondrial genome. As a consequence, cells treated with these drugs should be depleted of respiratory chain subunits encoded by the mitochondrial DNA. To confirm that the used concentrations of thiamphenicol and ethidium bromide extensively inhibit biogenesis of the respiratory chain complexes in HeLa cells we analysed the steady-state level of the mitochondrially encoded COX2 protein (cytochrome $c$ oxidase subunit 2). Additionally, in cells treated with ethidium bromide we analysed the mtDNA content and the steady-state levels of two mitochondrial transcripts: ND3 and 16S rRNA.

The results presented in Fig. 1A indicate that both thiamphenicol and ethidium bromide efficiently inhibit synthesis of the mtDNA-encoded proteins in the HeLa cell line. The level of the COX2 protein in cells treated with both inhibitors was below detection after 6 days of drug treatment. In the same time we observed a 10-fold decrease in the mtDNA content in cells treated with ethidium bromide (Fig. 1B) which is in full agreement with previously published data (Seidel-Rogol \& Shadel, 2002). The steady-state level of the ND3 transcript was undetectable using Northern blot analysis during the whole 6-day period of ethidium bromide treatment. We also observed a gradual decrease of the 16S rRNA steady-state level (Fig. 1C). $16 \mathrm{~S}$ rRNA content fell below $1 \%$ of the level in control cells after 6-days of drug treatment. These results indicate that the applied concentrations of the inhibitors efficiently inhibited expression of the mitochondrial genome.

\section{Ethidium bromide and thiamphenicol treatment af- fect bioenergetic metabolism of HeLa cells}

All 13 proteins encoded by the mitochondrial genome are subunits of the respiratory chain complexes. In order to measure how thiamphenicol and ethidium bromide influence the activity of the respiratory chain complexes in HeLa cells we analysed the cytochrome $c$ oxidase (COX) activity. Figure 2A shows that COX activity in cells treated with the inhibitors for 6 days was decreased by about $95 \%$ in comparison with the control cells. Cytochrome $c$ oxidase seems to be the control point of the overall respiratory chain activity and the oxidative phosphorylation process as COX activity was found to be correlated with cell respiration and ATP synthesis (Villani \& Attardi, 1997; D'Aurelio et al., 2001). Thus, around 20-fold COX activity reduction suggests that respiratory chain activity is significantly decreased during a 6-day period of the drug treatment.

As the respiratory chain activity decreases, HeLa cells activate the glycolytic pathway as an alternative source of the ATP. Data presented in Fig. 2B indicate a 2-fold increase in lactate production after 6 days of drug treatment. This result indicates that decreased ATP production by the mitochondria is at least partially compensated by enhanced glycolysis, an alternative source of ATP.

Increased lactate production is accompanied by a decrease in pyruvate production. Observed changes in lactate and pyruvate production led to an increase of the [lactate]:[pyruvate] ratio as shown in Fig. 2C. The [lactate]:[pyruvate] ratio in untreated HeLa cells is 17:1. Both thiamphenicol and ethidium bromide treatment lead to a significant increase of this ratio up to 250:1. The most pronounced increase is observed between the 4th and 6th day of the drug treatment. The relatively high [lactate]:[pyruvate] ratio on the 6th day is due to a very low, almost undetectable, level of pyruvate. Increased [lactate]:[pyruvate] ratio and lactate production indicate a defect in respiratory chain activity and are commonly used in diagnostics of mitochondrial disorders (Robinson, 1996). An increase of both these parameters in HeLa cells indicates that changes in the energy metabolism caused by the used inhibitors are comparable with changes observed in cells harbouring defects in the oxidative phosphorylation system.

\section{Thiamphenicol and ethidium bromide treatment results in reduced growth of HeLa cells}

One of the most energy consuming processes in the cell is cell division and DNA replication during the $S$ phase of the cell cycle. In order to investigate whether thiamphenicol or ethidium bromide treatment influences cell growth we analysed generation time of HeLa cells in two day intervals. The obtained results presented in Fig. 2D show a significant reduction of cell growth. The generation time of untreated HeLa cells was about 24 h. However, thiamphenicol and ethidium bromide treatment significantly influences the generation time of HeLa cells. The most pronounced changes were observed during the first two days of drug treatment. The average cell underwent only one division. Some recovery of cell growth was observed during the next 4 days. However, generation time (around $34 \mathrm{~h}$ ) was 

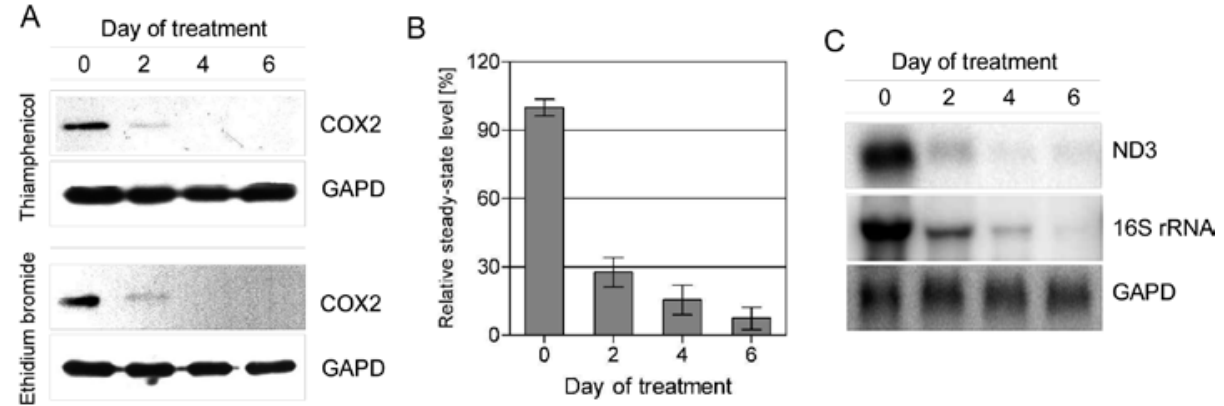

Figure 1. Expression of the mitochondrial genome in HeLa cells treated with thiamphenicol or ethidium bromide. (A) Steady-state level of the COX2 subunit encoded by the mitochondrial DNA assayed using Western blot analysis. Antibodies directed against glyceraldehyde-3-phosphate dehydrogenase (GAPD) were used for standardisation. (B) Steady-state level of the mtDNA in HeLa cells treated with ethidium bromide was analysed using real-time PCR technique. mtDNA copy number was standardized on the nuclear $\beta$-2-microtubulin gene. For better visualisation, the results were recalculated taking the steady-state level of the mtDNA at time point 0 as 100. Steady-state levels are presented as the value \pm standard deviation. (C) Steady-state levels of $16 \mathrm{~S}$ rRNA and ND3 mitochondrial transcripts in HeLa cells treated with ethidium bromide were assayed using Northern blot analysis. The transcript encoding GAPD was used for standardisation.

still longer when compared with control cells. Inhibition of HeLa cell growth indicates that increased glycolysis is not capable of fully compensating the reduced activity of the respiratory chain. The presented set of experiments shows that thiamphenicol or ethidium bromide treatment leading to decrease of respiratory chain activity results in metabolic stress of HeLa cells.

A

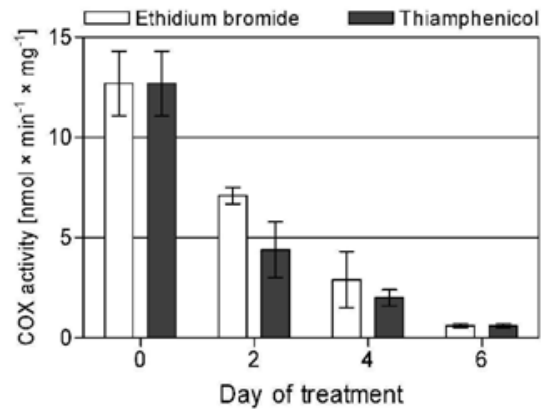

C

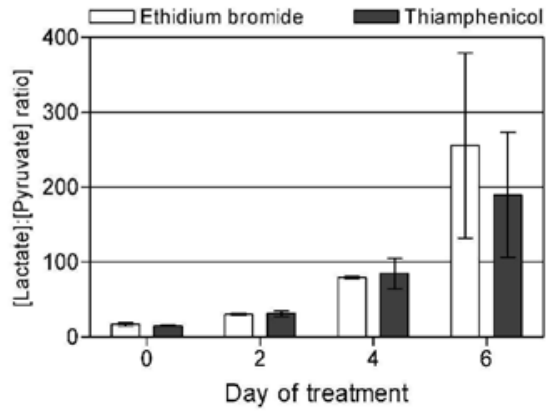

Energetic stress does not influence the steady-state levels of the NRF-1 and TFAM transcripts

NRF-1 plays a key role in regulation of the mitochondrial capacity for energy generation. It was shown that increased expression of the NRF-1 gene is necessary and sufficient to activate synthesis of $\delta$ aminolevulinate (ALA) synthase in HeLa cells ( $\mathrm{Li}$ et B

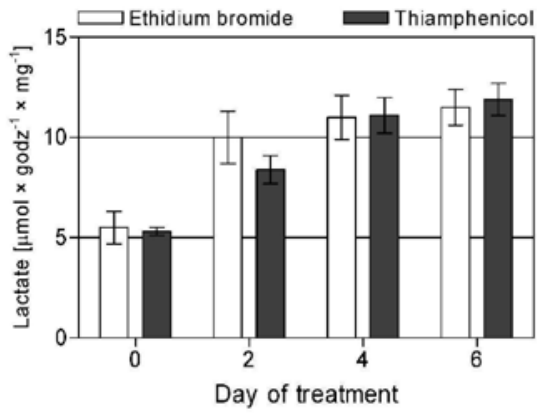

D

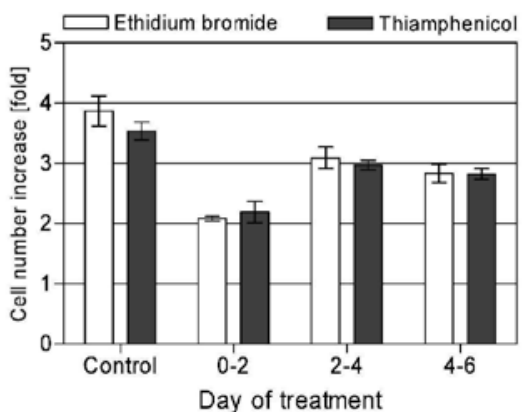

Figure 2. Bioenergetic metabolism of HeLa cells treated with thiamphenicol or ethidium bromide (A) Cytochrome $c$ oxidase activity. (B) Lactate production. (C) [lactate]:[pyruvate] ratio. (D) Growth of HeLa cells treated with thiamphenicol or ethidium bromide. Cytochrome $c$ oxidase activity was assayed using reduced cytochrome $c$ as the substrate. In order to analyse lactate and pyruvate production HeLa cells were incubated in PBS supplemented with $25 \mathrm{mM}$ glucose. The produced metabolites were assayed using spectrophotometric methods. Cell growth was analysed in two day intervals. HeLa cells were seeded into dishes at starting density of $2.5-4 \times 10^{4} \mathrm{cells} / \mathrm{cm}^{2}$. After two days cells were harvested and counted. Results are presented as the multiplicity of cell number increase. All results are presented as the value \pm standard deviation. 


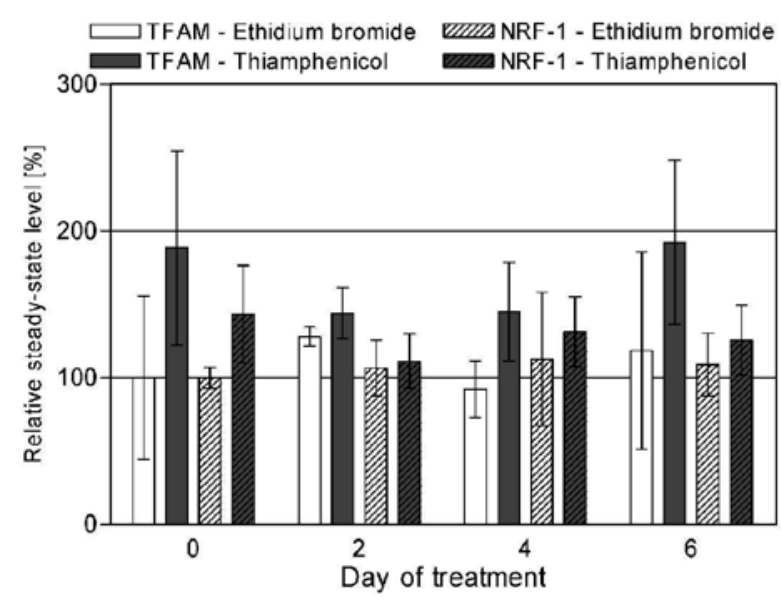

Figure 3. Relative steady-state levels of the TFAM and NRF-1 transcripts in HeLa cells treated with thiamphenicol or ethidium bromide.

Steady-state levels were analysed using real-time PCR technique and standardised on the GAPD transcript. For better visualisation of the results, the steady-state levels were recalculated taking the steady-state level of the appropriate transcript at 0 time point as 100 . Steady-state levels are presented as the value \pm standard deviation.

al., 1999). This enzyme is rate-limiting for the synthesis of heme, a crucial part of respiratory chain complexes. NRF-1 also regulates expression of the mitochondrial transcription factor A (TFAM) thus ensuring coordinated expression of both nuclear and mitochondrial genomes (Virbasius \& Scarpulla, 1994).

As increased levels of the NRF-1 and TFAM mRNAs may indicate enhanced mitochondrial biogenesis we investigated the steady-state levels of both these transcripts in HeLa cells treated with thiamphenicol and ethidium bromide. The results presented in Fig. 3 show that NRF-1 and TFAM transcripts are not elevated in cells treated with the inhibitors. Both transcripts were standardized in respect to the glyceraldehyde-3-phosphate dehydrogenase (GAPD) transcript. Expression of this transcript was shown to be stable in HeLa cells without functional respiratory chain complexes (Li et al., 1995). These results indicate that short-term inhibition of mitochondrial genome expression does not induce increased expression of TFAM and NRF-1 transcription factors.

Thiamphenicol and ethidium bromide treatment does not influence the citrate synthase activity and the steady-state levels of mitochondrial proteins encoded in the nucleus

In order to confirm the lack of enhanced mitochondrial biogenesis in HeLa cells with disrupted mtDNA expression we analysed steady-state levels of several mitochondrial proteins. Cytochrome $c$ and cytochrome $c$ oxidase 5a subunit (COX5a) are components of the respiratory chain. Hsp60 is a chaperone involved in the folding of the proteins imported into the mitochondria. As shown in Fig. 4A

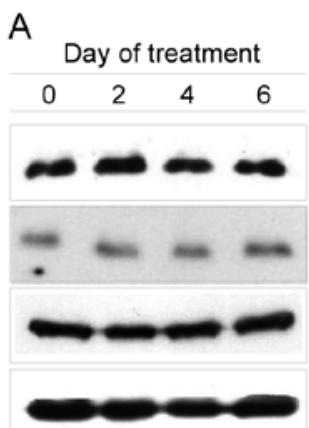

Thiamphenicol

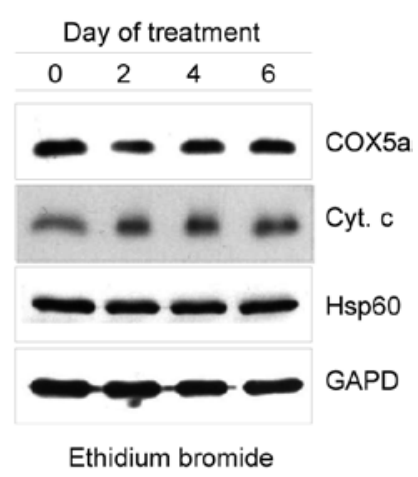

B

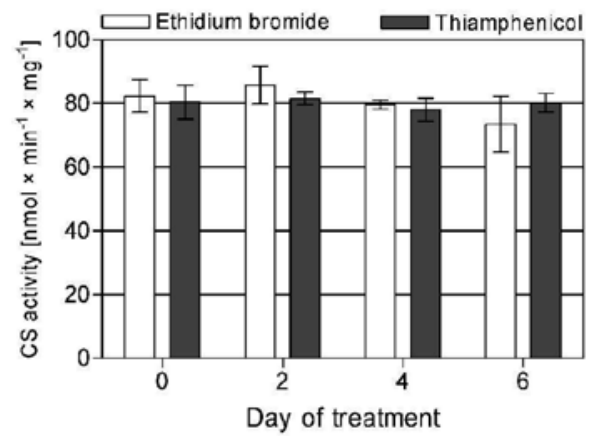

C

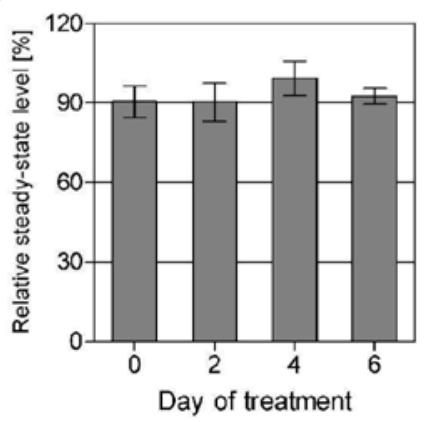

Figure 4. Mitochondrial protein levels, citrate synthase activity and mtDNA levels in thiamphenicol- or ethidium bromide treated cells.

(A) Steady-state level of mitochondrial proteins citrate synthase activity (value \pm standard deviation) (B) in HeLa cells treated with thiamphenicol or ethidium bromide and mtDNA steady-state level in HeLa cells treated with thiamphenicol (value \pm standard deviation) (C). Steady-state levels of the mitochondrial proteins were assayed using Western blot analysis. Anti-GAPD antibodies were used for standardisation. Citrate synthase activity was assayed according to standard procedures using acetyl-CoA and oxalacetate as substrates. Steady-state level of the mtDNA was analysed using Real Time PCR technique. mtDNA copy number was standardized on the nuclear gene of $\beta$ 2 -microtubulin. For better visualisation, the results were recalculated taking the steady-state level of the mtDNA at time point 0 as 100 . 
A

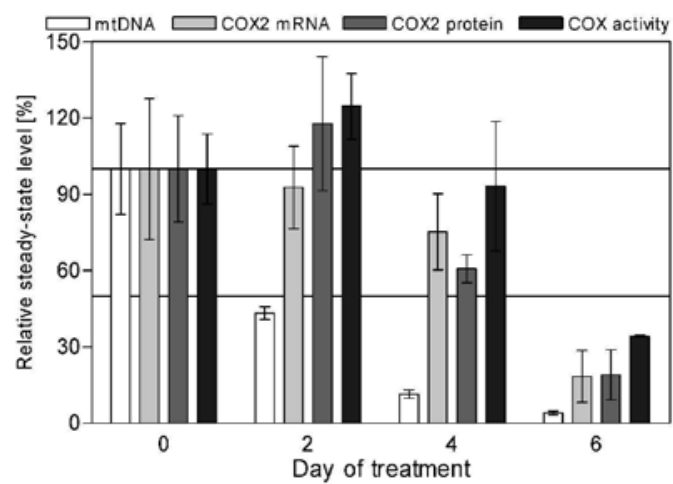

B

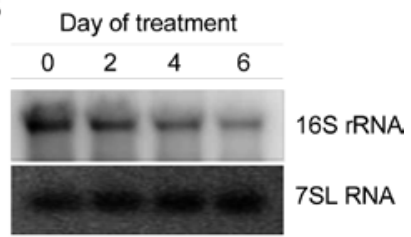

C

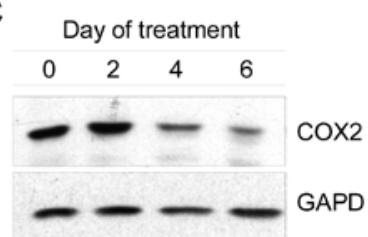

Figure 5. Expression of the mitochondrial genome in HeLa cells with depleted mitochondrial DNA.

mtDNA depletion was obtained by treatment with dideoxycytidine $(20 \mu \mathrm{M})$. (A) A graph presenting mtDNA, COX2 mRNA, COX2 protein steady-state levels and cytochrome $c$ oxidase (COX) activity. mtDNA and COX2 mRNA steadystate levels were assayed using real-time PCR and standardised on $\beta$-2-microtubulin single copy gene and GAPD transcript, respectively. All data are presented as the value \pm standard deviation. (B) Steady-state level of the 16S rRNA analysed using Northern blot analysis. (C) Exemplary Western blot analysis of the COX2 protein steady-state level presented on the graph.

no significant increase of the analysed proteins was observed in HeLa cells treated with thiamphenicol or ethidium bromide. Another indication confirming lack of enhanced mitochondrial biogenesis in HeLa cells with inhibited mtDNA expression was obtained after analysis of citrate synthase (CS) activity. The activity of this enzyme has been shown to be proportional to mitochondrial mass (Hoppeler, 1986). Data depicted in Fig. 4B indicate that citrate synthase activity is not affected during cell growth in the presence of thiamphenicol or ethidium bromide.

mtDNA content is not affected by thiamphenicol treatment

mtDNA steady-state levels may be increased in some patients showing clinical manifestations characteristic of mitochondrial disease (Bai et al., 2004) Thus, it may be suspected that energy deprivation caused by decreased activity of the respiratory chain may be compensated by overreplication of the mtDNA in order to ensure sufficient synthesis of respiratory chain subunits. In order to examine whether decreased activity of the respiratory chain in HeLa cells treated with thiamphenicol influences the mtDNA content we investigated the mtDNA steady-state levels in cells treated with the inhibitor for six days. Data presented in Fig. 4C indicate no changes in the mtDNA level in cells treated with thiamphenicol. This result shows that lack of mitochondrial translation does not lead to increase of the mtDNA copy number. Thus, in HeLa cells decreased expression of the mitochondrial genome is not compensated by increased mtDNA replication.
mtDNA depletion is compensated at the level of mt-RNA metabolism

The obtained results suggest that no increased biogenesis occurs in HeLa cells with disrupted mtDNA expression for the period of 6 days. In the next step we decided to investigate possible compensation mechanisms in the mitochondria using dideoxycytidine (ddC). This inhibitor in the used concentration prevents only mtDNA replication (Simpson et al., 1989), thus it is more specific than ethidium bromide which inhibits both replication and transcription. After 6 days of treatment with ddC the mtDNA was decreased to $4 \%$ of the level observed in control cells (Fig. 5A). This result shows that $\mathrm{ddC}$ is very effective in inhibition of mtDNA replication in HeLa cells.

At the same time we investigated steady-state levels of two mitochondrial transcripts: 16S rRNA and COX2. Both of them were reduced (Fig. 5A and $5 \mathrm{~B})$. However, decrease of the $16 \mathrm{~S}$ rRNA and COX2 steady-state levels appeared to be much less pronounced in comparison with mtDNA. For example on the 4th day of ddC treatment steady-state levels of both analysed transcripts were reduced by less than $40 \%$, despite a $90 \%$ reduction of the mtDNA level.

In the last step we analysed the steady-state level of the COX2 protein and cytochrome $c$ oxidase (COX) activity (Fig. 5A and 5C). We observed a slight increase of the steady-state level of the COX2 protein on the 2 nd day accompanied by increased activity of cytochrome $c$ oxidase. On the 4th day of ddC treatment COX activity was reduced less than $10 \%$ in spite of a $90 \%$ decrease of the mtDNA level. Thus, a 10-fold reduced mtDNA level resulted in 
an almost normal activity of cytochrome $c$ oxidase. We found that a statistically significant difference existed only between steady-state levels of mt-RNA and mtDNA during the analysed time period. There were no statistically significant differences between steady-state levels of COX2 mRNA, COX2 protein and COX activity. These results indicate that mitochondria in HeLa cells have the potential to compensate decreased mtDNA copy number at least during a relatively short time of inhibition of mtDNA replication. Significant compensation of mtDNA depletion is observed only at the level of mitochondrial RNA metabolism.

\section{DISCUSSION}

Thiamphenicol or ethidium bromide treatment leads to a bioenergetic shift in HeLa cells

Both ethidium bromide and thiamphenicol inhibit expression of the mitochondrial genome resulting in a lowered content of the mitochondrially encoded proteins and decreased activity of the respiratory chain. The content of COX2 protein after 6-day treatment of HeLa cells is not detectable by Western blot analysis. At the same time activity of cytochrome $c$ oxidase is decreased by $95 \%$. Lowered activity of the respiratory chain is linked to an increase in lactate production. This result indicates that lack of ATP synthesized in the oxidative phosphorylation process is compensated by increased glycolysis. Lactate production on the 6th day of drug treatment is increased over 2-fold. This result suggests that oxidative phosphorylation is responsible for about $50 \%$ of the ATP synthesis in HeLa cells.

In addition to glycolytic pathway activation, reduction of HeLa cell growth is observed. Generation time during the first two days of drug treatment is increased two-fold. At the same time the most pronounced increase of lactate production is observed. This observation suggests that HeLa cells shift from metabolism based on oxidative phosphorylation to metabolism based mostly on glycolysis. However, the increased activity of the glycolytic pathway seems to be insufficient for the compensation of the decrease in oxidative phosphorylation as cell division, a highly energetic process, is strongly suppressed.

The balance between the ratio of NADH and $\mathrm{NAD}^{+}$concentrations in the cytoplasm and the ratio of lactate and pyruvate concentrations is maintained due to lactate dehydrogenase. In cells with a nonfunctional respiratory chain the transport of electrons from NADH into the mitochondria is blocked.
Therefore $\mathrm{NAD}^{+}$is recovered by pyruvate oxidation resulting in an elevated [lactate]:[pyruvate] ratio. The [lactate]:[pyruvate] ratio in untreated HeLa cells is around 17:1. Treatment of HeLa cells with both thiamphenicol and ethidium bromide leads to significant elevation of this ratio up to 250:1.

\section{Short-term inhibition of the mitochondrial genome expression does not induce increased proliferation of mitochondria}

The obtained results showed that the energy metabolism of HeLa cells grown in the presence of thiamphenicol or ethidium bromide for 6 days was strongly affected. However, steady-state levels of NRF-1 and TFAM remained unchanged. These results are consistent with results obtained by Selwood et al. (2001), who have shown that an 11-day period of thiamphenicol treatment does not influence the level of TFAM protein and the level of active NRF-1 protein in the Hep G2 cell line.

Levels of several mitochondrial proteins selected for analysis appeared to be unaffected in HeLa cells treated with thiamphenicol or ethidium bromide. The activity of citrate synthase was also unchanged. As activity of this enzyme is correlated to the mass of mitochondria in the cell, this is a strong indication that proliferation of mitochondria is not enhanced during 6-day treatment of HeLa cells with thiamphenicol or ethidium bromide.

The obtained results indicate that short term inhibition of mitochondrial genome expression leading to significant decrease of respiratory chain activity does not induce mitochondrial biogenesis. Enhanced mitochondrial biogenesis (e.g. increased expression of NRF-1 and TFAM factors) was observed in $Q_{0}$ HeLa cells (Miranda et al., 1999) treated with ethidium bromide for a period of at least 70 passages. This observation indicates that induction of enhanced mitochondrial proliferation is time-dependent and a relatively long time is necessary to induce nuclear genome response to decreased expression of the mitochondrial genome.

This observation suggests that inhibition of mitochondrial genome expression and decreased activity of the respiratory chain resulting in depletion of ATP produced in the oxidative phosphorylation process are not direct signals inducing mitochondrial proliferation. Thus, increased biogenesis of mitochondria observed in tissues of patients harbouring mutations in mtDNA is rather due to long-term exposure of the cells to oxidative stress/abnormal $\mathrm{Ca}^{2+}$ homeostasis than to decreased expression of the mtDNA. Both increased $\mathrm{Ca}^{2+}$ levels in the cytoplasm and increased reactive oxygen species (ROS) have been documented in patients with defects in respiratory chain complexes. It has been also shown that both factors 
are capable of inducing increased mitochondrial proliferation (extensively reviewed by Lee \& Wei (2005)). However, this explanation suggests that increased mitochondrial proliferation may not be a programmed cellular response tending towards compensation of decreased respiratory chain activity, but rather is an element of pathology of the mitochondrial diseases. Stimulation of mitochondrial biogenesis by reactive oxygen species leads to proliferation of abnormal mitochondria, which produce more ROS. Additionally, elevated ROS production increases oxidative damage of the mitochondria and mtDNA what additionally elevates the ROS production. The presence of this positive feed-back, also described as a vicious cycle, could explain formation of "red ragged fibers” (RRFs) which are commonly observed in patients harbouring mtDNA mutations and characterized by extremely high mutation load and increased accumulation of abnormal mitochondria.

Compensatory mechanisms are present in the mitochondria

Depletion of the mtDNA obtained due to dideoxycytidine $(\mathrm{ddC})$ treatment allowed the investigation of possible compensatory effects in the mitochondria. The obtained results indicate that HeLa cells possess a great potential to compensate mtDNA depletion. After two days of dideoxycytidine treatment the amount of mtDNA is decreased by $57 \%$. However, the steady-state level of COX2 mRNA is lowered only by $7 \%$. The amount of COX2 protein and cytochrome $c$ oxidase activity is even about $20 \%$ higher than in control cells. After four days of ddC treatment the mtDNA level is $11 \%$, but the cytochrome $c$ oxidase activity is lowered only by $7 \%$ compared with control cells. A significant, $66 \%$ decrease of COX activity is observed after 6 days of ddC treatment, when mtDNA content is lowered to $4 \%$. The presented results extend and specify observations made by Pan-Zhou et al. (2000) who analysed effects of antiretroviral nucleotide analogues on mitochondrial function in Hep G2 cells. In that case application $10 \mu \mathrm{M}$ ddC resulted in $>90 \%$ decrease of mtDNA content. However, activities of respiratory chain complexes analysed on 6th day of ddC treatment were not affected.

Our results clearly show that the most pronounced compensation of mtDNA depletion is obtained at the level of mitochondrial RNA metabolism. During the four first days of ddC treatment the COX2 mRNA steady-state level remained almost unchanged and a significant decrease was observed only on the 6th day, when its level was still 4.5 -fold higher in relation to mtDNA content. The steadystate level of $16 \mathrm{~S}$ rRNA after 6 days of ddC treat- ment was $44 \%$ of the control, representing an 11-fold elevation in relation to mtDNA content.

The level of COX2 protein and COX activity after the first two days of ddC treatment was slightly higher as compared with control, but the differences are not statistically significant. As cytochrome $c$ oxidase is formed of subunits encoded both by mitochondrial and nuclear genomes, an increase of its activity may suggest that ddC treatment activates mitochondrial biogenesis. However, previous work of Pan-Zhou et al. (2000) on the Hep G2 cell line has shown that ddC does not influence citrate synthase activity. Moreover, it was shown in the same work that normal activities of respiratory chain complexes were maintained despite significant reduction in protein synthesis in the mitochondria. Thus, it cannot be excluded that respiratory chain activity is achieved by increased stability of the complexes. Increased stability of cytochrome $c$ oxidase would explain its slightly elevated activity as well as the elevated level of COX2 protein in HeLa cells after 2 days of ddC treatment.

mtDNA level does not limit expression of the mitochondrial genome

Thiamphenicol treatment does not influence the mtDNA copy number in HeLa cells. However, it has been previously shown that thiamphenicol treatment leads to increase of mitochondrial mRNA steady-state levels in Hep G2 cells in spite of unaffected mtDNA content (Chrzanowska-Lightowlers et al., 1994; Selwood et al., 2001). This increase is due to specific activation of RNA synthesis from $\mathrm{It}_{\mathrm{H} 2}$ initiation site in the $\mathrm{tRNA}^{\text {Phe }}$ gene, because levels of rRNA transcripts synthesized mostly from $\mathrm{It}_{\mathrm{H} 1}$ site are not increased. However, our previous work has shown that thiamphenicol treatment led to significant stabilisation of three mitochondrial transcripts: ND2, ND3 and cytochrome $b$ in HeLa cells (Piechota et al., 2006). Thus, elevated content of the mRNA transcripts may be due to the superposition of both increased transcription and stability of the individual transcripts.

The observations presented above indicate that mtDNA copy number is not the main factor limiting expression of the mitochondrial genome. In fact, Wiesner et al. (1992) found no relation between the oxidative capacity of a tissue and its content of mitochondrial DNA in rats and suggested that transcriptional and posttranscriptional mechanisms rather than gene dosage determine expression of the mitochondrial genome. Further investigations indicated that the rate limiting factor for transcription might be mitochondrial transcription factor $\mathrm{A}$ (Garstka et al., 1994). Our results with ddC are in agreement with observations made for rat tissues and indicate that transcription initiation rather than 
mtDNA copy number is a rate limiting factor for expression of the mitochondrial genome also in human cell lines.

\section{Acknowledgements}

This study was supported by the State Committee for Scientific Research (KBN) Grant 2 P05A 01726 and by Warsaw University Intramural Grant BW1636-41.

\section{REFERENCES}

Bai RK, Perng CL, Hsu CH, Wong LJ (2004) Quantitative PCR analysis of mitochondrial DNA content in patients with mitochondrial disease. Ann N Y Acad Sci 1011: 304-309.

Brandon MC, Lott MT, Nguyen KC, Spolim S, Navathe SB, Baldi P, Wallace DC (2005) MITOMAP: a human mitochondrial genome database-2004 update. Nucleic Acids Research 33(Database Issue): D611-613, URL: http:// www.mitomap.org.

Chrzanowska-Lightowlers ZM, Preiss T, Lightowlers RN (1994) Inhibition of mitochondrial protein synthesis promotes increased stability of nuclear-encoded respiratory gene transcripts. J Biol Chem 269: 27322-27328.

Dang CV, Semenza GL (1999) Oncogenic alterations of metabolism. Trends Biochem Sci 24: 68-72.

D'Aurelio M, Pallotti F, Barrientos A, Gajewski CD, Kwong JQ, Bruno C, Beal MF, Manfredi G (2001) In vivo regulation of oxidative phosphorylation in cells harboring a stop-codon mutation in mitochondrial DNA-encoded cytochrome $c$ oxidase subunit I. J Biol Chem 276: 4692546932.

Darley-Usmar VM, Rickwood D, Wilson MT (1987) Mitochondria, a practical approach. IRL Press, Washington.

Garstka HL, Facke M, Escribano JR, Wiesner RJ (1994) Stoichiometry of mitochondrial transcripts and regulation of gene expression by mitochondrial transcription factor A. Biochem Biophys Res Commun 200: 619-626.

Goffart S, Wiesner RJ (2003) Regulation and co-ordination of nuclear gene expression during mitochondrial biogenesis. Exp Physiol 88: 33-40.

Heddi A, Lestienne P, Wallace DC, Stepien G (1993) Mitochondrial DNA expression in mitochondrial myopathies and coordinated expression of nuclear genes involved in ATP production. J Biol Chem 268: 1215612163.

Heddi A, Stepien G, Benke PJ, Wallace DC (1999) Coordinate induction of energy gene expression in tissues of mitochondrial disease patients. J Biol Chem 274: 2296822976.

Hoppeler H (1986) Exercise-induced ultrastructural changes in skeletal muscle. Int J Sports Med 7: 187-204.

Kroon AM (1965) Protein synthesis in mitochondria. 3. On the effects of inhibitors on the incorporation of amino acids into protein by intact mitochondria and digitonin fractions. Biochim Biophys Acta 108: 275-284.

Lee HC, Wei YH (2005) Mitochondrial biogenesis and mitochondrial DNA maintenance of mammalian cells under oxidative stress. Int J Biochem Cell Biol 37: 822-834.

Leonard JV, Schapira AH (2000) Mitochondrial respiratory chain disorders I: mitochondrial DNA defects. Lancet 355: 299-304.
Li K, Neufer PD, Williams RS (1995) Nuclear responses to depletion of mitochondrial DNA in human cells. Am J Physiol 269: 265-270.

Li B, Holloszy JO, Semenkovich CF (1999) Respiratory uncoupling induces $\delta$-aminolevulinate synthase expression through a nuclear respiratory factor-1-dependent mechanism in HeLa cells. I Biol Chem 274: 1753417540.

McKenzie M, Liolitsa D, Hanna MG (2004) Mitochondrial disease: mutations and mechanisms. Neurochem Res 29: 589-600.

Miranda S, Foncea R, Guerrero J, Leighton F (1999) Oxidative stress and upregulation of mitochondrial biogenesis genes in mitochondrial DNA-depleted HeLa cells. Biochem Biophys Res Commun 258: 44-49.

Nisoli E, Clementi E, Paolucci C, Cozzi V, Tonello C, Sciorati $C$, Bracale $R$, Valerio A, Francolini $M$, Moncada S, Carruba MO (2003) Mitochondrial biogenesis in mammals: the role of endogenous nitric oxide. Science 299: 896-899.

Ozawa M, Nonaka I, Goto Y (1998) Single muscle fiber analysis in patients with 3243 mutation in mitochondrial DNA: comparison with the phenotype and the proportion of mutant genome. J Neurol Sci 159: 170-175.

Pan-Zhou XR, Cui L, Zhou XJ, Sommadossi JP, Darley-Usmar VM (2000) Differential effects of antiretroviral nucleoside analogs on mitochondrial function in HepG2 cells. Antimicrob Agents Chemother 44: 496-503.

Petruzzella V, Moraes CT, Sano MC, Bonilla E, DiMauro S, Schon EA (1994) Extremely high levels of mutant mtDNAs co-localize with cytochrome $c$ oxidase-negative ragged-red fibers in patients harboring a point mutation at nt 3243. Hum Mol Genet 3: 449-454.

Piechota J, Tomecki R, Gewartowski K, Szczesny R, Dmochowska A, Kudla M, Dybczynska L, Stepien PP, Bartnik E (2006) Differential stability of mitochondrial mRNA in HeLa cells. Acta Biochim Polon 53: 157-168.

Pillar TM, Seitz HJ (1997) Thyroid hormone and gene expression in the regulation of mitochondrial respiratory function. Eur I Endocrinol 136: 231-239.

Robinson BH (1996) Use of fibroblast and lymphoblast cultures for detection of respiratory chain defects. Methods Enzymol 264: 454-464.

Rotig A, Munnich A (2003) Genetic features of mitochondrial respiratory chain disorders. J Am Soc Nephrol 14: 2995-3007.

Sambrook J, Russel DW (2001) Molecular Cloning. A Laboratory Manual. Cold Spring Harbor Laboratory Press, Cold Spring Harbor, New York.

Scarpulla RC (1997) Nuclear control of respiratory chain expression in mammalian cells. J Bioenerg Biomembr 29: 109-119.

Scarpulla RC (2002) Nuclear activators and coactivators in mammalian mitochondrial biogenesis. Biochim Biophys Acta 1576: 1-14.

Scarpulla RC (2005) Nuclear control of respiratory gene expression in mammalian cells. J Cell Biochem 97: 673683.

Seidel-Rogol BL, Shadel GS (2002) Modulation of mitochondrial transcription in response to mtDNA depletion and repletion in HeLa cells. Nucleic Acids Res 30: 1929-1934.

Selwood SP, McGregor A, Lightowlers RN, Chrzanowska-Lightowlers ZM (2001) Inhibition of mitochondrial protein synthesis promotes autonomous regulation of mtDNA expression and generation of a new mitochondrial RNA species. FEBS Lett 494: 186-191.

Simpson MV, Chic CD, Keilbaugh SA, Lin TS, Prusoff WH (1989) Studies on the inhibition of mitochondrial DNA 
replication by $3^{\prime}$-azido-3'-deoxythymidine and other dideoxynucleoside analogs which inhibit HIV-1 replication. Biochem Pharmacol 38: 1033-1036.

Singh KK, Kulawiec M, Still I, Desouki MM, Geradts J, Matsui S-I (2005) Inter-genomic cross talk between mitochondria and the nucleus plays an important role in tumorigenesis. Gene 354: 140-146.

Skladal D, Halliday J, Thorburn DR (2003) Minimum birth prevalence of mitochondrial respiratory chain disorders in children. Brain 126: 1905-1912.

Stump CS, Short KR, Bigelow ML, Schimke JM, Nair KS (2003) Effect of insulin on human skeletal muscle mitochondrial ATP production, protein synthesis, and mRNA transcripts. Proc Natl Acad Sci USA 100: 79968001.

Tsiriyotis C, Spandidos DA, Sekeris CE (1997) The mitochondrion as a primary site of action of glucocorticoids: mitochondrial nucleotide sequences, showing similarity to hormone response elements, confer dexamethasone inducibility to chimaeric genes transfected in LATKcells. Biochem Biophys Res Commun 235: 349-354.

Vandesompele J, De Preter K, Pattyn F, Poppe B, Van Roy N, De Paepe A, Speleman F (2002) Accurate normalization of real-time quantitative RT-PCR data by geomet- ric averaging of multiple internal control genes. Genome Biol 3: RESEARCH0034.

Villani G, Attardi G (1997) In vivo control of respiration by cytochrome $c$ oxidase in wild-type and mitochondrial DNA mutation-carrying human cells. Proc Natl Acad Sci USA 94: 1166-1171.

Virbasius JV, Scarpulla RC (1994) Activation of the human mitochondrial transcription factor A gene by nuclear respiratory factors: a potential regulatory link between nuclear and mitochondrial gene expression in organelle biogenesis. Proc Natl Acad Sci USA 91: 1309-1313.

Wiesner RJ, Ruegg JC, Morano I (1992) Counting target molecules by exponential polymerase chain reaction: copy number of mitochondrial DNA in rat tissues. Biochem Biophys Res Commun 183: 553-559.

$\mathrm{Wu} \mathrm{H}$, Kanatous SB, Thurmond FA, Gallardo T, Isotani E, Bassel-Duby R, Williams RS (2002) Regulation of mitochondrial biogenesis in skeletal muscle by CaMK. Science 296: 349-352.

Zong H, Ren JM, Young LH, Pypaert M, Mu J, Birnbaum MJ, Shulman GI (2002) AMP kinase is required for mitochondrial biogenesis in skeletal muscle in response to chronic energy deprivation. Proc Natl Acad Sci USA 99: 15983-15987. 